\title{
Christiane Wiesenfeldt
}

\section{»Mediatrix nostra « - »Unsere Vermittlerin«: Marianische Topoi in Pierre de la Rues Messen für Margarete von Österreich}

Dass unter den habsburgisch-burgundischen Hofkomponisten um 1500 der aus Tournai stammende Pierre de la Rue einen, wenn nicht den herausragenden Platz einnimmt, ist kein Geheimnis. Ebenso ist bekannt, dass sein nach heutigem Kenntnistand 32 Titel umfassender Messenkatalog erst nach seiner Anstellung bei Hofe im Jahre 1492 entstand und daher offenbar mit den vielfältigen Aufgaben und Bedürfnissen der »Grande Chapelle« assoziiert ist. Wenig Aufmerksamkeit hat trotz des wachsenden Forschungsinteresses ${ }^{1}$ an la Rue sein Corpus der Marienmessen erregt ${ }^{2}$, dabei besetzen die insgesamt 13 Werke - eines gilt als verschollen - thematisch doch immerhin mehr als ein Drittel des Bestandes. Zugleich, und auch dies ist bislang nicht näher in Augenschein genommen worden, scheinen beinahe alle diese Messen in mehr oder weniger deutlichem Zusammenhang mit der Statthalterin der Niederlande, Margarete von Österreich, zu stehen, in deren Diensten sich la Rue ab

1 Hervorzuheben sind insbesondere die Arbeiten von Honey Meconi: Style and Authenticity in the Secular Music of Pierre de la Rue, Diss. Harvard University 1986, »Pierre de la Rue and Secular Music at the Court of Marguerite of Austria ", in: Jaarboek van het Vlaamse Centrum voor Oude Muziek 3 (1987), S. 49-58, »Free from the Crime of Venus: the Biography of Pierre de la Rue«, in: Revista de musicología 16 (1993), S. 2673-2683, »French Print Chansons and Pierre de la Rue. A Case Study in Authenticity«, in: Music in Renaissance Cities and Courts. Studies in Honor of Lewis Lockwood, hrsg. von Jesse Ann Owens und Anthony M. Cummings, Warren 1997, S. 187-213, Pierre de la Rue and musical life at the Habsbourg-Burgundian Court, Oxford 2002. Darüber hinaus erschienen zuletzt folgende Beiträge: J. Evan Kreider, »Pierre de la Rue's Incessament and its Musical Descendants«, in: From Ciconia to Sweelinck: donum natalicium Willem Elders, hrsg. von Albert Clement und Eric Jas, Amsterdam 1994, S. 167-178; Nigel Davison, »Absalom fili mi Reconsidered«, in: TVNM 46 (1996), S. 42-56; Willem Elders, »Number Symbolism in Some Cantus-Firmus Masses of Pierre de la Rue«, in: Jaarboek van het Vlaamse Centrum voor Oude Muziek 3 (1987), S. 59-68.

2 Gleiches gilt für die bislang kaum untersuchten Motetten. Hinzuweisen ist in dem Zusam menhang auf das von der Verfasserin und Wolfgang Fuhrmann organisierte Symposion zu Pierre de la Rues Motettenschaffen auf der Medieval é Renaissance Music Conference 2010, Royal Holloway University London, 5. bis 8. Juli 2010. Eine Publikation der Beiträge wird im Heft $1 / 2011$ der »Tonkunst« stattfinden. 
1508 offiziell befand, die er aber bereits bei zahlreichen früheren Gelegenheiten musikalisch bedient zu haben scheint.

Wenn im Folgenden versucht wird, Margarete von Österreichs marianisch-mariologisches Interesse auf den Gebieten von Kunst, Literatur und (privater wie offizieller) Frömmigkeit mit den Messkompositionen la Rues in Zusammenhang zu bringen, so können freilich nur ausgewählte Aspekte berührt werden. In einem deutlichen Missverhältnis stehen dabei einerseits die komplexe Materialfülle zur Marienverehrung Margarete von Österreichs, in die für den vorliegenden Zusammenhang allenfalls nur erste Schneisen geschlagen werden können ${ }^{3}$, und andererseits das weitgehend vage Feld der kompositorischen Anlassbindung, die - wo nicht durch Indizienverdichtung Substanz gewinnend - zumeist auf dem Niveau der Spekulation verbleiben muss. Nichtsdestotrotz sind die Messen sämtlich auf bedeutsame Ereignisse oder Kontexte beziehbar, was hier anzudeuten und damit weiteren Nachforschungen zu öffnen ist.

Ausgangspunkt der Überlegungen war zunächst die Beobachtung, dass mit der auffällig personifizierten Missa O gloriosa Margaretha eine für die betreffende Zeit ungewöhnliche Titelung vorlag: Ungewöhnlich nicht deswegen, weil der Herrschername titelbildend war - ein Usus spätestens seit Josquins Missa Hercules Dux Ferrariae -, sondern weil sich hier reale marianische Hymnenvorlage (»O gloriosa domina«) und reale, zumal weibliche Herrscherfigur zusammenfanden. Richtete sich anschließend der Blick auf das Marienmessen-Corpus insgesamt, so zeigte sich, dass nach aktuellem philologischen Kenntnisstand fast alle Werke nach 1508, d. h. nach dem offiziellen Engagement la Rues am Hofe Margaretes von Österreich entstanden und ihr somit vermutlich zuzuordnen sind. Für zwei frühere Werke sind darüber hinaus ebenso Bezugspunkte auszumachen: Überzeugungskraft gewinnen diese weniger in ihren biographischen und damit lückenhaften Kontexten als vielmehr entweder durch zeitlich und thematisch korrelierende Kunstwerke, die Margarete von Österreich erwarb bzw. beauftragte, oder als

3 Die Marienverehrung Margarete von Österreichs ist bislang keiner expliziten Untersuchung unterworfen worden, die sie berücksichtigenden Texte benennen sämtlich nur Aspekte des Gebiets, vgl. dazu insbesondere Dagmar Eichberger, Leben mit Kunst. Wirken mit Kunst (Burgundica V), Turnhout 2002, darin Teil 2: »Marienverehrung in Mecheln und Brou«, S. 207-228; Women of Distinction. Margaret of York, Margaret of Austria ( Ausstellungskatalog Mechelen, 17. September bis 18. Dezember 2005), hrsg. von Dagmar Eichberger, Leuven 2005, darin Kathryn M. Rudy, »Woman's Devotion in Mechelen«, S. 231-239; Ursula Tamussino: Margarete von Österreich. Diplomatin der Renaissance, Graz 1995, darin »Der Musenhof zu Mecheln«, S. 169-193. 
historisch-politische Ereignisse in Burgund, die eine musikalische Initiative la Rues nahelegen.

Eröffnet werden können so aus unterschiedlichen Perspektiven erste Einblicke in die ebenso intensiv wie individuell ausgeprägte Marienfrömmigkeit in der Hofhaltung Margaretes von Österreich, die den frömmigkeitsgeschichtlichen Horizont spannt, in dem sich la Rues auffällig viele Marienmessen verankern. ${ }^{4}$ Dass die Motivationen, denen die Marienverehrung Margaretes folgte, keineswegs nur religiös, sondern mindestens ebenso politisch grundiert waren, schärft das Profil der Musik im höfisch-repräsentativen Tableau der Künste umso mehr, nicht zuletzt, weil sich diese in der Regel öffentlich ereignete. Frömmigkeit diente hier nicht selten als Vehikel politischpersönlicher Selbstinszenierung, soll heißen: Die dem Marienglauben inhärente Massen- und Kultus-Wirkung nutzte Margarete von Österreich - abgesehen von ihrer eigenen, privaten und hier nicht infrage zu stellenden Gläubigkeit - als Katalysator ihrer politischen Präsenz. Salopp formuliert, schlug sie mit der Beauftragung, Präsentation und Pflege marianisch konnotierter Kunst zwei Fliegen mit einer Klappe.

Die folgenden Ausführungen sind in drei Abschnitte gegliedert: 1. ist ein kurzer Überblick über la Rues Marienmessen zu geben, wie er sich nach aktuellem Forschungsstand darstellt, 2. sollen die Marienverehrung Margarete von Österreichs und ihre damit verbundenen Intentionen anhand ausgewählter Beispiele erhellt werden und 3. sind die Marienmessen, soweit möglich, in den skizzierten Kontext der höfischen Frömmigkeitskultur einzupassen.

\section{Pierre de la Rues Marienmessen}

Ein einfacher Übertrag sämtlicher, in den Lexika und Monographien la Rues versammelter Marienmessen ergibt eine Liste von 24 Werken. Streicht man hingegen die Doppeltitel - außer in drei Fällen hat jedes Werk mindestens einen Alternativ-Titel in seiner Überlieferungsgeschichte erhalten -, so bleiben jene erwähnten 13 Messen stehen (vgl. die folgende Tabelle). Nach den Stemmata der Opera Omnia-Edition, die nur in einigen wenigen Punkten von Meconis Chronologie abweicht ${ }^{5}$, ergibt sich eine recht klare Ordnung.

4 Vgl. auch generell zur Hofhaltung im politischen und kulturellen Kontext Bertrand Schnerb: L'Etat bourguignon 1363-1477, Paris 1999.

5 Etwa die Position der Missa O gloriosa Margaretha wäre von Nr. 4 auf Nr. 9 versetzt, wenn man sie statt »vor 1510« (gemäß Opera Omnia) auf »vor 1516« datiert (gemäß Meconi, Pierre de la Rue and musical life [wie Anm. 1]) - der Unterschied resultiert aus der Umwertung der Frankfurter Quelle mit dem Text $\mathrm{O}$ gloriosa domina« als Nebenquelle bei 
In den meisten Fällen handelt es sich um Cantus firmus-paraphrasierende Messen, ihnen liegt entsprechend eine marianische Melodie unterschiedlichster Provenienz in allen Sätzen zugrunde. Aus dem formalen Rahmen fallen lediglich vier Werke: die Missa de septem doloribus (Nr. 7), die gleich mehrere, z. T. noch nicht identifizierte Vorlagen paraphrasiert, die Parodiemesse Ave sanctissima Maria (Nr. 8), der eine eigene Motette la Rues zugrunde liegt, die ein unbekanntes Ostinato nutzende Missa Sancta Dei Genitrix (Nr. 12) und das einzige marianische Choralordinarium Missa de Beata Virgine (Nr. 2).

\begin{tabular}{|c|c|c|c|c|c|}
\hline & Missa & Titel-Varianten & à & Vorlage(n) & $\begin{array}{l}\text { Datierung } \\
\text { (CMM 97) }\end{array}$ \\
\hline (1) & Assumpta est Maria & $\begin{array}{c}\text { De assumptione beate } \\
\text { marie }\end{array}$ & 4 & Antiphon & vor 1500 \\
\hline (2) & De beata Virgine & $\begin{array}{c}\text { De Domina } \\
\text { Salve sancta parens } \\
\text { Coronatum } \\
\end{array}$ & 4 & 5 Choräle & vor $1500-1503$ \\
\hline (3) & Sub tuum presidium & $\begin{array}{c}\text { In mi } \\
\text { Quarti toni }\end{array}$ & 4 & Antiphon & vor $1504 / 05$ \\
\hline (4) & Ogloriosa domina & $\begin{array}{c}\text { Ogloriosa Margaretha } \\
\text { Quatuor vocum }\end{array}$ & 4 & Hymnus & vor 1510 \\
\hline$(5)$ & Ave Maria & - & $4-5$ & Antiphon & vor $1511-1514$ \\
\hline (6) & Conceptio tua & - & 5 & $\begin{array}{l}\text { (Magnifikat-) } \\
\text { Antiphon }\end{array}$ & vor $1511-1515$ \\
\hline (7) & De septem doloribus & $\begin{array}{c}\text { De doloribus Marie } \\
\text { virginis } \\
\text { Dolores gloriose } \\
\text { recolentes } \\
\text { Quinque vocum de do- } \\
\text { loribus } \\
\end{array}$ & 5 & diverse & vor 1512 \\
\hline (8) & Ave sanctissima Maria & $\begin{array}{c}\text { De beata Virgine } \\
\text { Sex Vocum } \\
\text { Deus meus eripe me }\end{array}$ & 6 & eigene Motette & vor 1512 \\
\hline (9) & Ista est speciosa & $\begin{array}{c}\text { De sanctissima virgine } \\
\text { Maria } \\
\text { Quinque vocum }\end{array}$ & 5 & Antiphon & vor 1512 \\
\hline (10) & De Virginibus & $\begin{array}{c}\text { O quam pulchra } \\
\text { Pulchra es }\end{array}$ & 4 & Alleluiavers & $\begin{array}{c}\text { vor } 1513 \text { oder } \\
1516\end{array}$ \\
\hline (11) & Inviolata & Omnes peccaverunt & 4 & Sequenz & vor 1516 \\
\hline$(12)$ & Sancta Dei genitrix & Miserere mei Deus & 4 & Ostinato & vor 1521 \\
\hline- & Mediatrix nostra & - & - & - & - \\
\hline
\end{tabular}

Tabelle: Pierre de la Rues Marienmessen

Meconi, die die Handschrift mit dem Text $\gg \mathrm{O}$ gloriosa Margaretha« aus der Alamire-Werkstatt als Hauptquelle begreift (heute in Montserrat). Abweichungen ergeben sich gleichfalls bei der Brüsseler Quelle mit der Missa Ista est speciosa (hier an 8. bzw. 9. Position), die Meconi mit »nach 1521 «als posthum datiert, die Opera Omnia-Edition allerdings schon auf $»$ vor 1512« (plausibel angelehnt u. a. an den Census Catalogue). Vgl. Pierre de la Rue: Opera omnia, hrsg. von Nigel St. John Davison, J. Evan Kreider und T. Herman Keahey in 11 Bänden, die Marienmessen in den Bänden CMM 97, 1-6. 


\section{Margarete von Österreichs Marienverehrung}

Marienverehrung spielte in der Dynastie der Valois seit etwa 1400 eine große Rolle, legt man allein die in den Inventaren der Regentin dokumentierten gemalten und plastischen Portraits zugrunde, die enge Familienangehörige in der Anbetung Marias zeigen ${ }^{6}$ - angefangen bei Philipp dem Guten über Isabella von Portugal, Karl dem Kühnen, Isabella von Bourbon hin zu Margarete von Österreichs Vater Maximilian und ihrem ersten Mann Juan von Kastilien sowie dem Neffen Karl V. ${ }^{7}$ Mit letzteren ließ sich Margarete von Österreich u. a. gemeinsam in der Anbetung Marias darstellen. Ihre zahlreichen religiös konnotierten Portraits, die sie vor und während ihrer Re gentschaft ab 1507 in Auftrag gab, lassen sich verschiedenen Darstellungs modi und Motivgruppen zuordnen. ${ }^{8}$

Primäres Identifikationsmerkmal Margaretes von Österreich auf vielen religiösen, politischen oder religiös-politischen Portraits ist ihr Witwen-Habit, das sie nach dem Tod ihres dritten Mannes Philibert von Savoyen nicht mehr abgelegt hat: eine herzförmige, schlichte weiße Haube und eine Halskrause (vgl. Abbildung 1).

Derart präsentiert sie sich auch im Rahmen dynastischer Genealogien, etwa im Rahmen jener für Karl V. ${ }^{9}$ Gleichzeitig beginnt sie, ihr politisches Motto »Fortune - Infortune - Fort une« (»Glück - Unglück - stark allein«) verstärkt zu inszenieren, es findet sich in vielgestaltiger Form wieder, wie etwa auf einer Münzprägung, die die schicksalsbesiegende Stärke der »filia Maximiliani« akzentuiert (vgl. Abbildung 2).

6 Eichberger, Leben mit Kunst. Wirken mit Kunst (wie Anm. 3), S. 207.

7 Ebd., S. 194 (zu Maximilian), 44 (zu Juan de Kastilien) sowie zu beiden S. 208.

8 Vgl. zur Portraitsammlung Margaretes Dagmar Eichberger und Lisa Beaven: »Family Members and Political Allies: The Portrait Collection of Margaret of Austria «, in: The Art Bulletin 77 (1995), Heft 2, S. 225-248; Andrea G. Pearson: »Margaret of Austria's Devotional portrait Dipytchs «, in: Woman's Arts Journal 22 (2001), Heft 2, S. 19-24.

9 La genealogie de l'empereur Charles Vme de ce nom [...], 1527-1530; Quelle: Paris BNF, ms. fr. 5616. 


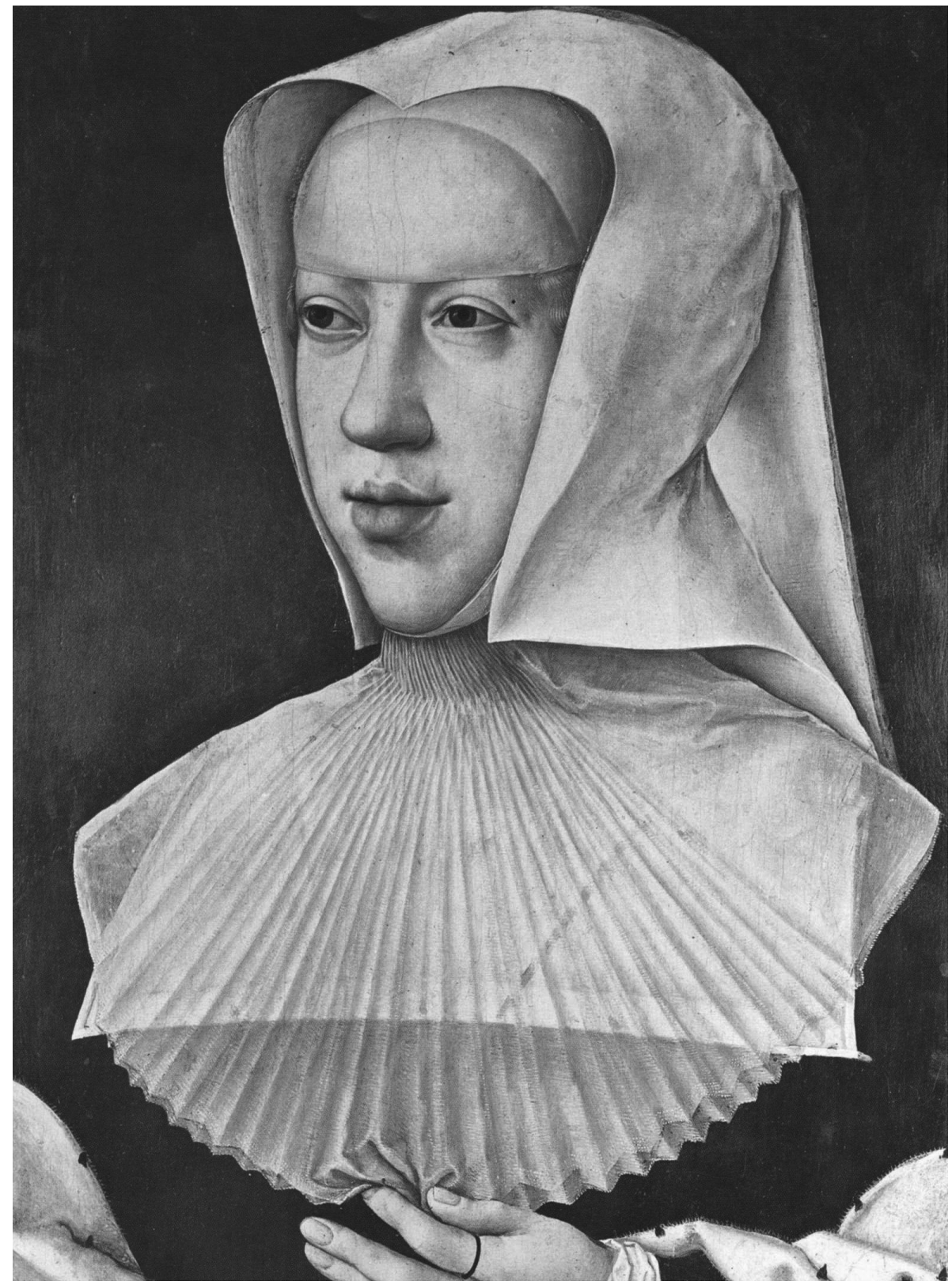

Abbildung 1: Margarete von Österreich in Witwen-Habit (typische Darstellung ab 1504), Bernard van Orley, nach 1518, Quelle: Bourg-en-Bresse, Monastére Royal de Brou, inv. no. 975, $16 \mathrm{AB}$ 

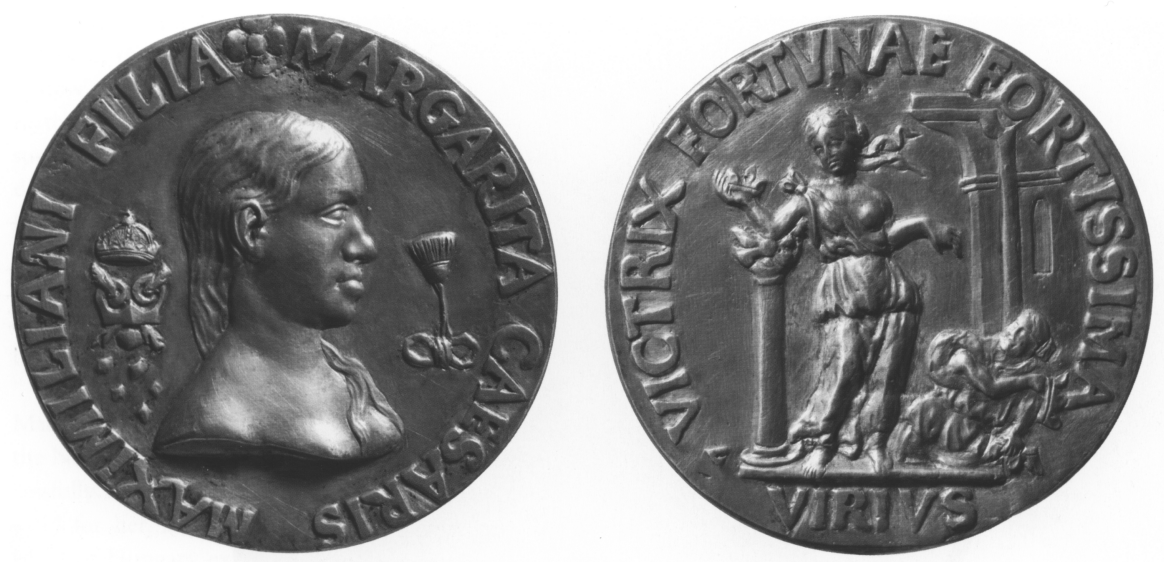

Abbildung 2: Portraitmedaille mit Margarete von Österreich und Fortuna, ca. 1505 Quelle: Kunsthistorisches Museum Wien

Derartige politische Tugenden ließen sich aber besonders eindrücklich im christologischen Kontext, insbesondere der Passion, herausstellen, worauf Margarete von Österreich gleich mehrfach zurückgegriffen hat. So lässt sie sich etwa allegorisch als Justitia in eine Kreuzigungsszene versetzen ${ }^{10}$, wodurch ihre judikatorische Gewalt als Herrscherin in einen milden, mitfühlenden Gestus gekleidet wird und sie zugleich - wie so häufig in blauem Gewand - neben Maria die wichtigste Frauenfigur des Tableaus darstellt. Ihre humanitäre, dem Volk gegenüber wohltätige Haltung ließ die Regentin häufig in gleichem Motiv demonstrieren, etwa als der neben Maria der Kreuzigung beiwohnende Caritas. ${ }^{11}$

Während das allegorische Portrait schon darauf verweist, dass religiöse Motive politischen Ambitionen und Inszenierungen als Justitia oder Caritas offenbar optimale Folien boten, indem mythologische Allegorien im Gewand weltlicher Realfiguren als Interpreten oder Kommentatoren religiöser Szenen auftreten, wird im sakralen Identifikations- oder auch Kryptoportrait eine Überblendung der Heiligenfigur mit dem weltlichen Herrscher bzw. Auftraggeber angestrebt. Dabei liegt in der Regel zunächst einmal der jeweilige namensgebende oder zumindest namensverwandte Schutzpatron auf der

10 Vgl. z. B. Margarete als Justitia im Rahmen einer Kreuzigungsszene von Olivier de la Marche: Le paremant et triumphe des dames, Tapesterie, 2. Viertel des 16. Jh., Paris BNF, ms. fr. 25431.

11 Vgl. z. B. Margarete als Caritas, Kreuzigungsszene von Bernard van Orley, ca. 1523/24, Öl auf Holz, Rotterdam, Museum Bijmans Van Beuningen, inv. no. 1629. 
Hand: im Falle Margaretes von Österreich die Heilige Margarete, die sie schon früh (ab 1497) und sodann regelmäßig mimt. ${ }^{12}$ Bis zur Gestaltung ihres Grabmonumentes in Mechelen zieht sich dieser Topos durch ihre Kunstsammlung (vgl. Abbildung 3).

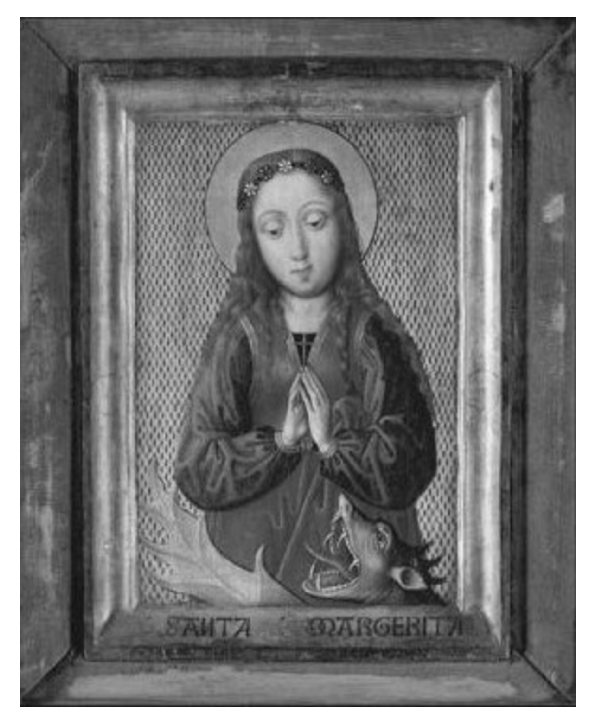

Abbildung 3: Margarete von Österreich als HI. Margarete Michel Sittow, ca. 1497, Quelle: Privatbesitz

Die Figur Maria spielt sodann - wie am Beispiel der Kreuzigungsszenen und der ähnlichen Gewandung angedeutet - eine zentrale Rolle für Margarete von Österreich, selbst wenn bislang kein Kryptoportrait als Maria zweifelsfrei nachgewiesen werden kann. Dies wäre vermutlich auch als Anmaßung ausgelegt worden. Für den Zusammenhang ist hingegen nicht unwesentlich, dass Margarete von Österreich jedes ihrer Gemächer mit mindestens einem Marienbild geschmückt hatte, zahlreiche Marienreliquien besaß, und ihre etwa 100 religiösen Bücher eine deutliche Tendenz zu marianisch-mariologischen Themenkreisen aufwiesen, demnach Marienviten ebenso umfassten wie Marienlegenden, Andachtsbücher, Marienoffizien usw. ${ }^{13}$ Überdies war Margarete von Österreich seit den 1490er Jahren Mitglied der frommen Bru-

12 Michel Sittow, Margarete von Österreich als Hl. Margarete, Teil eines Diyptychons mit Juan von Kastilien als Hl. Johannes auf der anderen Seite (verschollen), ca. 1497, Öl auf Holz, 28 x 20,3 cm, in Privatbesitz (Auktion Christie's New York: Mittwoch, 25. Mai 2005), Abb. unter http://www.artnet.de/Artists/LotDetailPage.aspx?lot_id=22404A507 097C2A7D83E3E408332DC70, 9. Januar 2010.

13 Zur Bibliothek vgl. u. a. Marcel Françon, Gh. de Boom: »Activité Littéraire à la Cour de Marguerite d'Autriche«, in: The Modern Language Journal 16 (1931), Heft 3, S. 249-251. 
derschaft der sieben Schmerzen Mariens in Brügge und engagierte sich 1517 für die Kirchweihe eines neu gegründeten Frauenkonvents ebenfalls zu den Sieben Schmerzen. Auch zu diesem wichtigen marianischen Topos sind Kunstwerke in ihrem Besitz zu benennen. ${ }^{14}$

Zwar mag Margarete von Österreichs marianisches Engagement im Kontext höfischer Religiosität des frühen 16. Jahrhunderts, die zumal gegenreformatorisch ausgerichtet war und auch die finanziellen Mittel besaß, um der Kunst ausgiebig und nachhaltig zu frönen, kein Einzel- oder Sonderfall sein. Bemerkenswert ist das Engagement aber dann, wenn man die politische Intention nicht aus den Augen verliert, die die kunstsinnige Marienfrömmigkeit stets grundierte.

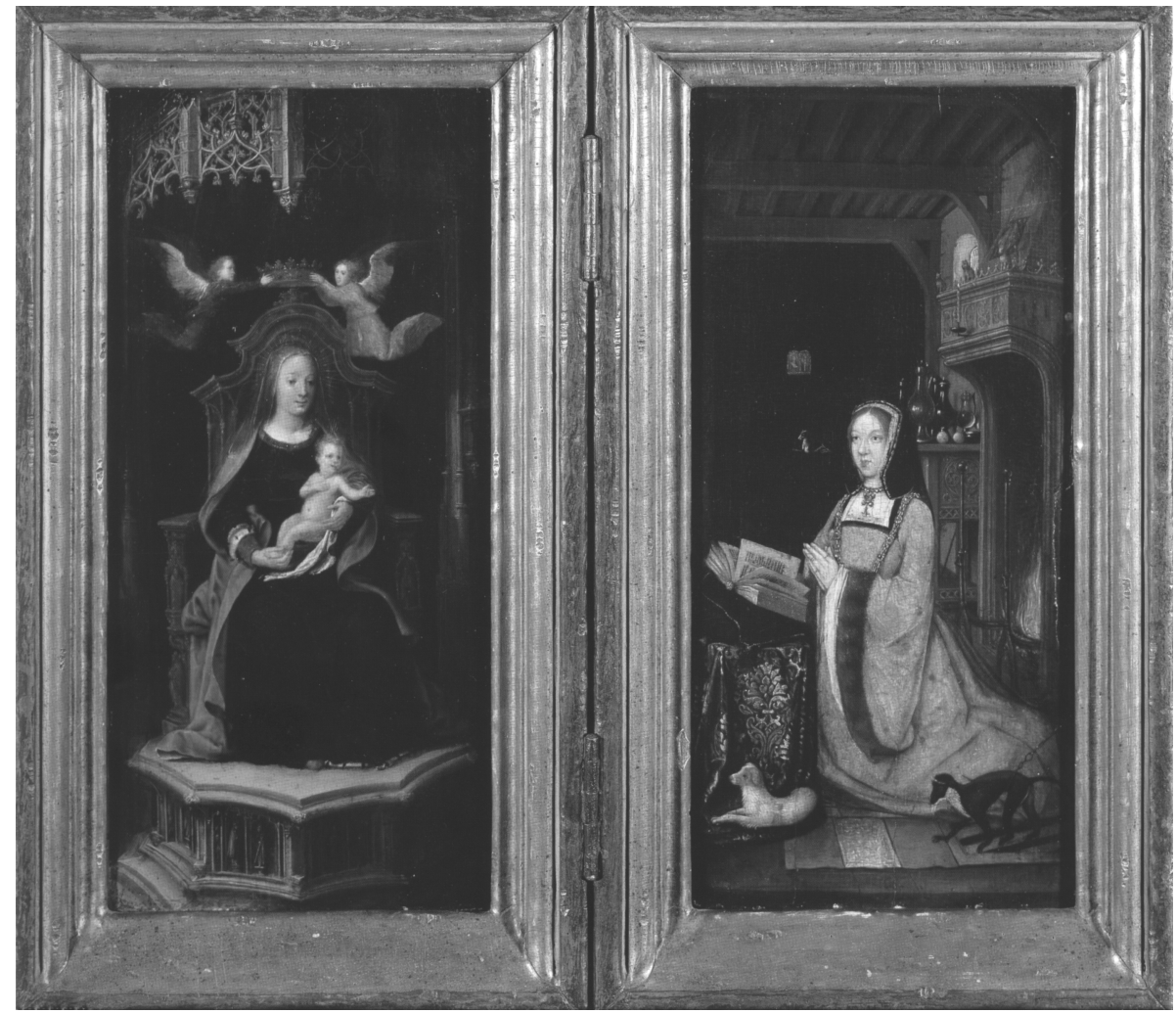

Abb. 4: Diptychon: Maria und Margerete von Österreich, »Meister von 1499«, ca. 1501-1504, Quelle: Ghent, Museum voor Schone Kunsten, inv. no. 1973A

14 Vgl. dazu insbes. den erwähnten Ausstellungskatalog Women of Distinction (wie Anm. 3). 
Ein erstes Diptychon noch vor ihrer Witwenzeit (sie trägt noch keine weiße Haube und höfische Gewänder) zeigt Margarete von Österreich in kniender Andachtspose vor der gekrönten, leicht erhöht thronenden Maria auf der linken Seite: eine zwar fürbittende Pose, die aber ansonsten nur von männlichen Herrschern eingenommen wird (vgl. Abbildung 4). Allein die Szenenwahl kann man folglich als politisches Statement lesen, bekannt aus fast identischen Darstellungen mit Maximilian I. oder Philipp dem Guten. Bereits als Herzogin von Savoyen war Margarete von Österreich bestrebt, ihre politischen Ambitionen auch qua symbolträchtiger marianischer Anbetungspose zu äußern.

Nicht wesentlich anders sind die Miniaturen zu Pierre de la Rues »Ave sanctissima Maria «-Motette in dem berühmten, von Margarete von Österreich beauftragten musikalischen Album von ca. 1516-1523 ausgearbeitet. ${ }^{15}$ Die lateinische Marienmotette steht bekanntermaßen an exponierter Position der Handschrift, gefolgt von zumeist weltlicher Musik. In den einleitenden Miniaturen trägt die bereits als Witwe zu erkennende Margarete von Österreich einerseits nun einen Herrscherinnen-Mantel und agiert folglich bereits als burgundische Regentin, deren Wappen mit ihren Insignien zumal auf gleicher Seite prangt. Anderseits spricht sie Maria nun direkt an und richtet an sie die Worte $»$ Memento mei« (»Gedenke meiner $«$ ), entstammend der Schlusszeile des »Ave Maria«: »O Mater Dei, memento mei. Amen«. Hier wird die weltliche Herrscherin nicht als stumme, gläubige und einige Stufen tiefer kniende Bittende, sondern bereits als die religiöse Mittlerfunktion Marias voraussetzende und selbstbewusst ansprechende (um nicht zu sagen fordernde) Machthaberin , auf Augenhöhe ${ }^{6}$ präsentiert (das Empfinden einer >Augenhöhe< stellt sich nicht zuletzt durch die Notenlinien zur ersten Zeile $»$ Ave sanctissima Maria« ein). Beide Figuren sind zudem eingefasst von ähnlichen Bildprogrammen mit persönlichen Schmuckstücken der Regentin ebenso wie dem Blumen-Motiv der Margerite.

Ein drittes, heute nicht mehr erhaltenes, dafür mehrfach dokumentiertes Beispiel schließlich zeigt Margarete von Österreich und das von Maria ihr entgegen gehaltene Christuskind in einem unmittelbaren Dialog. ${ }^{16}$ Endgültig - und nicht nur wie im Alamire-Manuskript über gegenüberliegende Seiten

15 Album der Margarete von Österreich, ca. 1516-1523, Brüssel, Bibliothéque Royale, ms 228.

16 Diptychon: Maria und Margerete, (nach) Bernard van Orley, 1518/20, Roubaix, Musée des Beaux Arts, Collection Lepoutre, Bild mittlerweile verschollen; Nachweis lediglich im Ausstellungskatalog: Exposition organisee par la Ville de Bourg-en-Bresse, en hommage a Marguerite d'Autriche, fondatrice de Brou (1480-1530), 1. Juni bis 15. Juli 1958, Bourg-en-Bresse 1958, S. 19-20. 
angedeutet - ist die fürbittende Position zugunsten einer Vis-à-vis-Ebene aufgegeben. Darüber hinaus sitzen sich beide Figuren im realen Fürstinnenzimmer gegenüber, und dies zumal am selben Tisch, der lediglich durch das Format des Diptychon geteilt ist. Das von Maria Margarete von Österreich entgegengehaltene Christuskind erhält vom Portraitisten das Wort »Veni« in den Mund gelegt, das ebenso als »Komm’ zu mir« (invitatorisch) wie »Ich bin gekommen« (inkarnatorisch) übersetzt werden kann. Margarete von Österreichs Antwort »Placet« / »Es gefällt mir« ist sodann Reaktion und nicht Aktion, eine Teilhabe an einem Dialog und keine monologisierende Anbetung mehr. Marias Vermittlung zwischen Mensch und Gott, ihre Aufgabe als »mediatrix«, ist hier der direkten Kommunikation zwischen gläubiger Chris tin und Gott (= Christus) gewichen; Margarete von Österreich kann nunmehr, gleich Maria, direkt mit Christus kommunizieren. So verschieben sich gegenüber den vorherigen beiden Diptychen die Akzente noch einmal deutlich, und die zumal eher nonnenhafte, gegenüber Maria schlichte Tracht der Regentin auf dem verlorenen Portrait mag eine künstlerische Lizenz an das vertrauliche, in seiner Lokalisation beinahe alltägliche Arrangement mit seiner durchaus progressiven Aussage sein.

Als Albrecht Dürer 1521 Mechelen besuchte, erhielt er die Gelegenheit, Margarete von Österreichs Privatsammlung zu besichtigen, die - da ihm gegenüber das Privileg der Besichtigung betont wurde - offenbar nicht allgemein zugänglich, sondern besonderen Gästen vorbehalten war. Insofern mögen manche der marianisch konnotierten Machtbekundungen der Regentin weniger der Repräsentation in der Öffentlichkeit denn der privaten Selbstvergewisserung gedient haben. Andere wiederum - wie das musikalische Album oder weitere, für jedermann sichtbar am Hofe platzierte oder Dritten dedizierte Darstellungen hingegen - mögen durchaus einer breiteren Aufmerksamkeit ausgesetzt und dies auch kalkuliert haben. Ob die Darstellungen, die besonders eng an Maria heranrücken, Margarete von Österreich nun aber in ihrer staatlichen Repräsentation dienten oder nicht: Fest steht, dass sich in ihnen eine über die fromm-devote Andachtsgeste weit hinausgehende Quasi-Identifikation mit Maria abzeichnete, die bis in die Adaption der marianischen $»$ mediatrix $\ll-$ Kompetenz hineinragt.

Die Motivation hierfür resultiert freilich kaum aus dem Bedürfnis nach religiöser Progressivität oder gar Anmaßung, sondern aus einer sich gleich aus mehreren Aspekten speisenden Identifikation der Regentin mit der Figur der tugendhaften, von Gott erwählten Mittlerin (und nicht zuletzt Frau) Maria. Inwieweit Margarete von Österreichs Ersatz-Mutterrolle für Karl V. hier hineinspielt, für den sie sich gleichermaßen als vormundschaftliche Hüterin 
wie vermittelnde Bereiterin einer weitaus bedeutenderen, kommenden Generation sah, muss Spekulation bleiben; denkbar ist es hingegen schon.

\section{Pierre de la Rues Marienmessen und Margarete von Österreichs Marienfrömmigkeit}

Mit la Rues Marienmessen trat die Marienfrömmigkeit Margarete von Österreichs nun gleichermaßen in ein anderes Medium ein wie überhaupt an die Öffentlichkeit. Offenkundig ist, dass manche dieser Werke zu repräsentativen Zwecken Verwendung gefunden haben. Nach dem Blick auf die Kunstsammlung verwundert nun weder die ungewöhnlich große Anzahl der marianischen Kompositionen mehr, noch der Sonderfall der Missa O gloriosa Margaretha, die beinahe als musikalisches Äquivalent zum zuletzt beschriebenen Diptychon wirkt, indem sich nun die »Domina [Maria] « zur $»[$ Domina] Margarete w wandelt. Selbst der Titel der verschollenen Missa Mediatrix nostra greift diesen identifikatorischen Topos auf, indem die aus dem Rosenkranzgebet an Maria gerichtete Anrufung »unsere Vermittlerin« zugleich als Ehrentitel der in nicht wenigen diplomatischen Verhandlungen äußerst erfolgreich vermittelnden Regentin gelesen werden kann: Vergegenwärtigt man sich ihre wichtigsten staatspolitischen Kunstgriffe zu Zeiten la Rues die Liga von Cambrai (1508), die Heilige Liga gegen Frankreich (1511) oder der Vertrag von Lille (1513) -, so wären bereits einige mögliche Anknüpfungspunkte zur Datierung der Messe gegeben, was allerdings aufgrund der vermissten Quelle obsolet ist. ${ }^{17}$ Dies lässt sich hingegen für andere Marienmessen aus der oben gezeigten Werkliste versuchen, wobei hier nur ausgewählte Werke und Aspekte zu Sprache kommen können, die anhand weiterer Quellen- und Dokumentensichtungen zu konkretisieren wären.

Geht man von der in der Opera Omnia-Edition aufgestellten Datierung aus, so ist jene Missa O gloriosa domina bzw. Margaretha die erste Messe, die la Rue als Hofkomponist Margaretes von Österreich vorlegte. Dass die frühere Quelle (datiert vor 1510) noch den hymnischen Originaltext »Domina« verwendet, muss nicht bedeuten, dass die sodann im Alamire-Prachtband von vor 1516 bezeugte Umwidmung $\mathrm{zu} »$ Margaretha« nicht schon früher

17 Denkbar ist, dass Missa Mediatrix nostra ein Alternativtitel der Missa Sub tuum praesidium ist, deren Text eine derartige Zeile enthält: »Sub tuum praesidium confugimus, sancta dei Genetrix. Nostras deprecationes ne despicias in necessitatibus nostris. Sed a periculis cunctis libera nos semper. Virgo gloriosa et benedicta, Domina nostra, mediatrix nostra, advocata nostra. Tuo Filio nos reconcilia, tuo Filio nos commenda, tuo Filio nos repraesenta. Amen.« 
bestand, da beide Quellen zumal auf eine - heute nicht nachweisbare Hauptquelle zurückgehen. Keineswegs abwegig ist, dass die Messe bereits 1507 oder 1508 entstand, etwa anlässlich Margarete von Österreichs Übernahme der Regentschaft am 18. März 1507, zum Zeitpunkt der Begräbnisfeierlichkeiten zum Tode Philipps I. in Mechelen am 18. bis 19. Juli 1507, denen la Rue beiwohnte und wo womöglich erste Überlegungen zu seiner Anstellung formuliert wurden, da sein Dienstherr ja soeben verstorben war, oder spätestens zum Juni 1508, zu dem die erste Gehaltszahlung für la Rue dokumentiert ist. ${ }^{18}$

Die an dritter Stelle rangierende Missa Sub tuum presidium (vor 1504/05) scheint zunächst außen vor zu stehen. Hier ließe sich allerdings im Zusammenhang mit der Nr. 2, der Missa de Beata Virgine, eine These formulieren, die zugleich beide Messen in das unmittelbare Umfeld der Regentin rückt. Von März bis August 1503 ist a Rue als Mitglied der Kapelle hilipps I. in Bourg-en-Bresse in Savoy bei Margarete von Österreich und ihrem Mann Philibert von Savoyen sowie in unmittelbarem lokalen Umfeld in Lyon nachweisbar. Aus exakt dieser Zeit stammt das erwähnte erste Diptychon Marga retes von Österreich in Anbetung Marias. Nur wenige Monate später - im Oktober 1503 - geht a Rues erstes Messbuch bei etrucci in den Druck, allen vorangestellt die erwähnte Missa de Beata Virgine. Mit einer dort ebenfalls enthaltenen Missa L'homme armé (vermutlich gemünzt auf den Dienstherren hilipp I.) sowie einer offenbar durch die soeben absolvierten spanischen Aufenthalte inspirierten Missa Nunca fue pena maior ist das Buch deutlich dem königlichen Hause zugeneigt, und die Eröffnung des Messbuches mit einer der soeben besuchten Schwester des Dienstherren thematisch assoziierten marianischen Komposition erscheint vor diesem Hintergrund plausibel. Überhaupt wäre das gesamte Messbuch als Ehrengabe an die habsburgische Familie zu lesen ${ }^{19}$ : Die an zweiter Stelle stehende Missa Puer natus nämlich könnte sich auf hilipps Sohn Ferdinand I. beziehen, der am 10. März 1503, also nur zwei Wochen vor der Visite in Savoyen, in Spanien zur Welt kam.

Die Missa Sub tuum presidium an dritter Stelle ist offenbar nach der Beata Virgine entstanden - hier stellt sich der oder die Bittende Sub tuum presidium, unter den Schutz Marias. Wie bekannt ist, wurde Margarete von Österreich im September 1504 überraschenderweise das zweite Mal Witwe, legte ihren dargestellten Habit an und beschloss, nicht mehr zu heiraten, sondern

18 Vgl. im Einzelnen die Dokumentenaufstellung und die biographische Skizze bei Meconi, Pierre de la Rue and musical life (wie Anm. 1).

19 Damit mögen die la Rue im Dezember 1503 zugesprochenen Pfründe in Namur in Zusammenhang stehen, vgl. Meconi, Pierre de la Rue and musical life (wie Anm. 1), S. 250. 
sich ausschließlich öffentlichen, wohltätigen Aufgaben zuzuwenden. Pierre de la Rue erreichte Ghent im November 1504, weitere Zahlungen an ihn aus Nijmegen, Arnheim, Brüssel usw. sind in den Folgemonaten bis zu seiner zweiten Abreise nach Spanien nachweisbar. Am 8. und 10. November 1504 ist la Rue sogar vor Ort in Mechelen bei Margarete von Österreich bezeugt. Auch hier wiederum ließe sich vermuten, dass die Regentin in inspirierendem Zusammenhang mit dieser gattungshistorisch bis dato solitären Marienmesse steht, die inhaltlich und zeitlich mit ihrer persönlichen Situation aufs Deutlichste korreliert.

Zur Missa de septem doloribus - nach der bisherigen Zählung der Marienmessen ausgerechnet die Nr. 7 - kann sodann angenommen werden, dass sie in Zusammenhang mit der gleichnamigen Bruderschaft steht, in der sich Margarete von Österreich langjährig engagierte. ${ }^{20}$ Nähere Untersuchungen hierzu stehen noch aus. Da im gesamten 16. Jahrhundert keine einzige andere Marienmesse dieses Titels und Themas überliefert ist, läge es nahe, für die Suche nach den verwendeten, bislang unidentifizierten melodischen Vorlagen auf die Materialien der Bruderschaft zurückzugreifen. Bekannt aus Briefen an die Äbtissin ist zudem, dass Margarete von Österreich zeitweilig vorhatte, ihren Lebensabend im Kloster der Bruderschaft zu verbringen: Sie ließ dort eigens ein Wohnhaus anbauen, was sie später aber nicht mehr in Anspruch nahm. Ein abschließender Hinweis auf die Fünfstimmigkeit mag sein, dass Margarete von Österreich nachweislich ein goldgerahmtes Tafelbild mit der Darstellung der Fünf Schmerzens Mariens besaß (heute verschollen), eine zeittypische Reduktion des Themas, die weite Verbreitung fand. Anhand des erhaltenen Buches der Bruderschaft im Alten Archiv in Brüssel wäre zu prüfen, ob die Messe womöglich gar erst zur Kirchweihe der Septem Doloribus 1517 entstand. Vor dem Hintergrund ihrer komplexen, materialintensiven und anspruchsvollen kompositorischen Faktur, die sie ohnehin in die Nähe der späten Messen rückt, wäre dies durchaus denkbar.

Womöglich steht die letzte Missa Sancta Dei genitrix (Nr. 12) ebenso Pate für Margarete von Österreichs Wiedererlangung der Macht im Jahre 1518, die sie aufgrund von innerpolitischen Intrigen und einer verfrüht ausgesprochenen Regierungsbefähigung Karls V. 1515 bis 1517 kurzfristig abzugeben

20 Eine andere Interpretation wäre womöglich, dass Margarete zur Zeit la Rues sieben wichtige Umbrüche und Todeserfahrungen in ihrer Familie erleben musste, angefangen vom Tod der Mutter (1482), der Annullierung der Ehe mit Karl VIII. (1491), die erste Witwenschaft (1497) mit der Totgeburt des einzigen Kindes (im selben Jahr), den Tod des zweiten Mannes (1504), den Tod des Bruders (1506) und den Tod der Stiefmutter (1510). Ob diese Ereignisse mit dem Thema überein zu bringen sind, bleibt freilich offen. 
hatte, wie auch in Zusammenhang mit dem letzten erwähnten, ebenfalls in diese Zeit zu datierenden Diptychon, auf dem Margarete von Österreich in den Dialog mit Christus tritt und damit zugleich ein Selbstbewusstsein zeigt, das seine Plastizität und Rigorosität womöglich der machtpolitischen Situation schuldet. Dass das der Messe zugrunde liegende, bislang unidentifizierte Ostinato sieben Noten - also zugleich die marianische Symbolzahl 7 sowie die sieben Silben des Namens »Mar-ga-ri-ta Aus-tri-a« oder auch ihres Mottos »Fortune / Infortune / Fort une - umfasst, verwundert wenig. Es ist darüber hinaus denkbar, dass es sich hier um ein soggetto cavato für Margarete von Österreich handelt. Obwohl bislang nicht gelungen ist, es zu identifizieren, scheiden zumindest sämtliche bekannten Marienmelodien als Vorlage aus.

Abgesehen von kontextuellen Aspekten der Werkentstehung, die Sujet, Titel sowie die Auswahl, Erfindung und Kombination der gewählten musikalischen Vorlagen einschließen, ist abschließend zu fragen, inwiefern sich darüber hinaus womöglich eine Akzentuierung marianischer (und damit >margaretischer $<$ ) Sujets in den Messen selbst finden lässt. Im Gegensatz zu gängigen Modellen der Herrschermesse, die den Namen des Regenten direkt zitieren, können hier lediglich marianische Topoi die Brücke zur Identifikation mit der in vielen Fällen sicherlich beauftragenden oder implizierten Widmungsträgerin schlagen. Da das Messformular Maria lediglich im Credo integriert - in der Zeile »ex Maria virgine « -, bleibt kaum Raum für symbolische Akzentuierung in >klassischer Form, wie der plakativen Herausstellung eines Namens, Mottos oder eines auf den zu Ehrenden gemünzten Textabschnitts. La Rue bedient sich hier verschiedener Hilfsmittel: In der Missa Ista est speciosa etwa wird die Antiphon »Ista est speciosa inter filias Jerusalem« als Cantus planus präsentiert und bleibt damit großflächig gleichermaßen >gewichtig< wie formgebend. Hingegen werden in der Missa de septem doloribus im 1. Tenor durchweg verschiedene Texte kompiliert, die sämtlich marianischen Themenkreisen entstammen und zu einem komplexen Textkorpus verschmolzen werden, dessen intertextuelle Charakteristika und Intentionen über seine Zugehörigkeit zur »Septem Doloribus«-Thematik hinaus bislang nicht untersucht sind. Ihre Fixierung auf psalmodische Tonrepetitionen legt ihre Herkunft aus Mariengebeten nahe, und tatsächlich lassen sich einige der Texte, wie der Psalter »Trenosa compassio« (im Christe) oder das dem »Stabat Mater« verwandte Gebet »Ferit gladius«, identifizieren. Im Agnus der Missa Ave Sanctissima Maria schließlich wird in den Oberstimmen ein neuer marianischer, bislang unidentifizierter Cantus firmus $\gg \mathrm{O}$ dulcis 
amica Dei« verwendet, und das Werk schließt obendrein nicht mit einem Agnus Dei II oder III, sondern mit einer Motette über den Text des Dankes hymnus $\gg$ Te decet laus«, die die gesamte Konstruktion in die Nähe der Motetti Missales rückt. Weitere Beispiele ließen sich ergänzen.

Fest steht bereits nach diesen ersten Annäherungen, dass die Marienmessen la Rues von ihrem regionalen und damit persönlichen Bezugspunkt Margarete von Österreich nicht zu trennen sind. Ihre Entstehungskontexte, aber auch ihre inneren Strukturen in Zukunft näher an die expansive und intensive Marienfrömmigkeit der Regentin in allen ihren Facetten heranzurücken als bislang geschehen, verspricht lohnende Ergebnisse. Dass diese ungewöhnliche Form der Herrscherinnenmesse nicht personifiziert, sondern mit Ausnahme der Missa O gloriosa Margaretha - über den allegorischen Umweg der marianischen Identifikation realisiert wurde, nimmt ihr kaum die Bedeutung, wenn auch sicherlich ihre Fasslichkeit. Es mag aber eben diese intellektuelle, elitär durchwirkte Subtilität und Selbstbezüglichkeit gewesen sein, die die fromme, gebildete und kunstsinnige Margarete von Österreich fasziniert haben mag. 